\title{
Anti-Neutrophil Elastase Defense of the Normal Human Respiratory Epithelial Surface Provided by the Secretory Leukoprotease Inhibitor
}

\author{
Claus Vogelmeier," Richard C. Hubbard," Gerald A. Fells," Hans-Peter Schnebli," \\ Robert C. Thompson," Hans Fritz," and Ronald G. Crystal* \\ *Pulmonary Branch, National Heart, Lung, and Blood Institute, National Institutes of Health, Bethesda, Maryland 20892, \\ ${ }^{\ddagger}$ Ciba-Geigy AG, Basel, Switzerland; 'Synergen, Boulder, Colorado; and "Department of Clinical Chemistry \\ and Clinical Biochemistry, University of Munich, West-Germany
}

\begin{abstract}
Secretory leukoprotease inhibitor (SLPI), a 12-kD nonglycosylated serine antiprotease with a high capacity for inhibiting neutrophil elastase (NE), is produced by cells of mucosal surfaces including the human lung. The molar concentrations of SLPI in total respiratory tract epithelial lining fluid (ELF) were $56 \pm 10 \%$ that of $\alpha 1$-antitrypsin, suggesting SLPI may be more important for the anti-NE protection of the pulmonary epithelial surface than previously thought. However, evaluation demonstrated that SLPI in respiratory ELF was only onethird functional. Studies aerosolizing recombinant SLPI (rSLPI) to sheep demonstrated that in the short term, neither aerosolization and alveolar deposition nor the lavage procedure inactivated the SLPI molecule. In vitro studies with rSLPI demonstrated that exposure to oxidants did not modify the form of the molecule, while exposure to oxidants and NE caused the molecule to be cleaved from 12 to $8 \mathrm{kD}$. Consistent with this, evaluation of SLPI in lavage fluid of individuals with cystic fibrosis (a condition with oxidants and NE on the respiratory epithelium) showed that the SLPI was degraded. However, evaluation of SLPI in normal ELF by molecular sieve analysis and Western analysis demonstrated an intact 12-kD molecule, suggesting that the partial inactivation of SLPI in normals in vivo is not because it is complexed to $\mathrm{NE}$ or exposed to oxidants + NE. Together, these observations demonstrate that SLPI is present in large amounts in respiratory ELF, but since the majority of the SLPI is inactive, it likely does not play a significant role in protecting the normal respiratory epithelium, except perhaps in the upper airways where the levels of SLPI are the highest. (J. Clin. Invest. 1991. 87:482-488.) Key words: $\alpha 1-$ antitrypsin • antiprotease $\bullet$ bronchoalveolar lavage
\end{abstract}

\section{Introduction}

Secretory leukoprotease inhibitor (SLPI), ${ }^{1}$ a $12-\mathrm{kD}$ nonglycosylated, single chain, 107 residue serine antiprotease, is pro-

Address correspondence and reprint requests to Pulmonary Branch, Building 10, Room 6D03, National Institutes of Health, Bethesda, MD 20892.

Received for publication 18 April 1990 and in revised form $13 \mathrm{Au}$ gust 1990

1. Abbreviations used in this paper: $\mathrm{r} \alpha 1 \mathrm{AT}$, recombinant $\alpha 1$-antitrypsin; ELF, epithelial lining fluid; NE, neutrophil elastase; PPE, porcine pancreatic elastase; SLPI, secretory leukoprotease inhibitor.

The Journal of Clinical Investigation, Inc.

Volume 87, February 1991, 482-488 duced by cells of mucosal surfaces and found in the corresponding epithelial lining fluids (1-5). SLPI inhibits several serine proteases, but with an association rate constant for neutrophil elastase (NE) of $\sim 10^{\chi_{1}} \mathrm{M}^{-1} \mathrm{~s}^{-1}$, its most likely principal physiologic function is to provide protection of local epithelial surfaces against NE (6). In the human lung, SLPI is observed in serous cells of submucosal tracheal and bronchial glands and in non-ciliated cells of the bronchial and bronchiolar epithelium, suggesting the major role of SLPI is to provide anti-NE protection for the tracheobronchial tree (7-9).

Although SLPI is clearly present on the respiratory epithelial surface (10-13), its form and function in lung epithelial lining fluid (ELF) is not well understood, nor is it clear whether it plays a role in the anti-NE protective screen of the pulmonary epithelium of normal individuals. The purpose of this study was to evaluate the amount and function of SLPI in lung ELF of healthy nonsmokers. To do so, we have capitalized on the ability to produce pure, functional SLPI by recombinant methods, permitting the development of assays that accurately quantify the amount and function of SLPI. Interestingly, the data demonstrate that while SLPI is present in significant amounts in normal respiratory ELF, the majority of the SLPI molecules in ELF are inactive.

\section{Methods}

Study population. The study population included 17 normal, nonsmoking individuals ( 9 female, 8 male, age $37 \pm 3 \mathrm{yr}$ ) (All data are presented as mean \pm standard error of the mean; all statistical comparisons were made using the two-tailed Student's $t$ test). All had normal lung function, chest radiographs, and no history of lung disease. Bronchoalveolar lavage demonstrated cell differentials typical for normal nonsmokers, including: alveolar macrophages $81 \pm 3 \%$, lymphocytes $17 \pm 3 \%$, neutrophils $1.8 \pm 0.4 \%$, and eosinophils $<1 \%$. As a control to determine the status of SLPI in vivo in circumstances where there is a massive burden of neutrophil elastase and oxidants on the pulmonary epithelial surface, two individuals with cystic fibrosis ( 2 males, 20 and $27 \mathrm{yr}$ ) were evaluated. Both had severe lung involvement (forced expiratory volume in $1 \mathrm{~s}, 59$ and $36 \%$ predicted, respectively). The cell differential in the bronchoalveolar lavage of these individuals showed a marked increase in the number of neutrophils ( 75 and $66 \%$ ).

Epithelial lining fluid. Routine bronchoalveolar lavage was performed in 11 of the normals and in both individuals with cystic fibrosis by instilling five aliquots of $20 \mathrm{ml}$ each of sterile $0.9 \% \mathrm{NaCl}$ in three different lobes and pooling all of the recovered fluid. Sequential bronchoalveolar lavage in normal individuals was performed by the infusion of a $20-\mathrm{ml}$ aliquot of normal saline followed by its immediate aspiration before instillation of any further fluid. This procedure was carried out five times in succession; three lobes were assayed in this manner. Each of the numbered aliquots from the lobes were pooled, and the fluid analysed. Dwell times were $<20 \mathrm{~s}$ per aliquot. The volume of ELF in the lavage samples was determined by the urea method (14). 
ELISA. SLPI, $\alpha 1$-antitrypsin ( $\alpha 1 \mathrm{AT})$ and albumin levels were quantified with specific double antibody sandwich ELISA. All assays were done in duplicate. In the SLPI ELISA (15), recombinant SLPI (rSLPI) identical to the naturally occurring SLPI, served as standard. The rSLPI was produced by Escherchia coli under the direction of a synthetic SLPI cDNA $(16,17)$. The cells expressing rSLPI were resuspended and passed through a pressure homogenizer and the lysate centrifuged and applied to an ion exchanger. Fractions containing rSLPI were subjected to a refolding process (18) and further purified by ion exchange and hydrophobic interaction chromatography. The purity of the rSLPI was $>99 \%$ as established by SDS/polyacrylamide gel electrophoresis and HPLC. The rSLPI standard was quantified spectrophotometrically based on an extinction coefficient of $0.8(0.1 \%$ solution, wavelength $275 \mathrm{~nm}$ ). The $\alpha 1$-antitrypsin ( $\alpha 1 \mathrm{AT}$ ) ELISA was carried out using a pure normal human plasma $\alpha 1 \mathrm{AT}$ standard $(19,20)$. For the albumin ELISA, the standard was a commercial human serum albumin preparation (Calbiochem-Behring Corp., San Diego, CA).

Evaluation of the form and function of SLPI. Evaluation of the ability of SLPI in lung to inhibit NE is complicated by the presence of large amounts of $\alpha 1 \mathrm{AT}$ in ELF. With available methods and antibodies, positive selection affinity purification of SLPI, in a manner analogous to methods we have used to purify $\alpha 1 \mathrm{AT}$ (20) from lavage fluid was not feasible. However, several alternative strategies were successful in evaluating the form and function of SLPI in respiratory ELF.

First, capitalizing on the knowledge that SLPI inhibits NE but not porcine pancreatic elastase (PPE) (10), the ability of lavage fluid to inhibit both NE and PPE was evaluated and the difference in lavage fluid [(anti-NE)-(anti-PPE)] capacity was compared with the amount of SLPI as determined by ELISA. Since the [(anti-NE)-(anti-PPE)] difference eliminates the contribution of $\alpha 1 \mathrm{AT}$ to the inhibition of NE, the ratio of [(anti-NE)-(anti-PPE)] to the amount of SLPI present reflects the functional activity of SLPI. To accomplish this, the ability of lavage fluid to inhibit NE was assessed (in triplicate) by titrating increasing amounts of the fluid against a fixed amount of a standard human NE (Elastin Products, Pacific, MO) with known activity determined as previously described (20). The remaining NE activity in the samples was determined using the NE specific substrate methoxy-succinyl-alanyl-alanyl-prolyl-valyl-nitroanilide (Sigma Chemical Co., St. Louis, MO) assessed spectrophotometrically at $410 \mathrm{~nm}$. The anti-PPE capacity of the lavage samples was determined in the same fashion as the anti-NE capacity (see above). The PPE standard for this assay (Calbiochem-Behring Corp.) was titrated in a fashion similar to that of NE, using the substrate succinyl-alanyl-alanyl-alanyl-nitroanilide (Calbiochem-Behring Corp.) (21).

Second, capitalizing on the ability to remove $\alpha 1 \mathrm{AT}$ from lavage fluid by affinity chromatography (20), the anti-NE capacity, anti-PPE capacity, SLPI level, and $\alpha 1$ AT level was evaluated in normal lavage fluid before and after anti- $\alpha 1 \mathrm{AT}$ affinity chromatography. To insure comparability between samples before and after chromatography, the samples were also evaluated for albumin, a natural constituent of lavage fluid that is not removed by anti- $\alpha$ 1AT affinity chromatography (20). After chromatographic removal of $\alpha 1 \mathrm{AT}$, the ratio of the anti-NE capacity to the amount of SLPI reflects the proportion of the SLPI that is functional as an inhibitor of NE.

Third, to insure that the method of obtaining SLPI from the respiratory epithelial surface (i.e., lavage) did not influence the amount and function of SLPI, rSLPI was aerosolized to sheep (a procedure known not to modify the molecule) $(20,22)$, the sheep lavaged to recover the SLPI, and the fluid evaluated for the amount and function of rSLPI. This was done using two approaches. To verify that no SLPI was lost during the recovery process by lavage, a known amount of rSLPI was aerosolized to sheep $(n=4,33 \pm 1 \mathrm{~kg})$ simultaneously with recombinant $\alpha 1 \mathrm{AT}$ ( $\alpha 1 \mathrm{AT}$; molar ratio of rSLPI to r $\alpha 1 \mathrm{AT}$ that was aerosolized $=3.75$ ) and the rSLPI and r $\alpha 1 \mathrm{AT}$ levels were assessed in bronchoalveolar lavage $1 \mathrm{~h}$ later using the SLPI ELISA and the $\alpha 1$ AT ELISA as previously described (23). To evaluate if the function of SLPI was inactivated by the lavage procedure, rSLPI $(50 \mathrm{mg})$ was aerosolized to sheep $(n=6,34 \pm 3 \mathrm{~kg})$. The concentration of rSLPI in lavage fluid $1 \mathrm{~h}$ post- aerosol was compared with the increase of the anti-NE capacity in the lavage fluid, i.e., a 1:1 increase would signify no loss in rSLPI by the recovery process.

Fourth, to investigate if part of the SLPI in normal bronchoalveolar lavage fluid was complexed by $\mathrm{NE}$, size exclusion high performance liquid chromatography was performed. Normal bronchoalveolar lavage fluid was concentrated 50 -fold by positive pressure filtration (YM5 membrane; Amicon Corp., Danvers, MA) and sized by HPLC (System Gold; Beckman Instruments Inc., Berkeley, CA) with a silica based molecular sieve (TSK 2000; Beckman Instruments, Inc.). The column fractions were collected and analyzed for SLPI using the specific ELISA. As standard, rSLPI was incubated with NE $\left(30 \mathrm{~min}, 25^{\circ} \mathrm{C}\right.$; molar ratio rSLPI/NE 2:1), chromatographed as above, and the fractions analyzed for SLPI.

Finally, to evaluate the possibility that some SLPI in normal ELF was oxidized and thus rendered nonfunctional as an inhibitor of NE, the form of SLPI in lavage fluid was evaluated by SDS/polyacrylamide gels and Western analysis using an anti-SLPI antibody and compared with SLPI known to be oxidized. For comparison, several forms of oxidized SLPI were evaluated, including: (a) rSLPI that had been incubated with $\mathrm{N}$-chlorosuccinimide (Sigma Chemical Co.) in a molar ratio of $400: 1$ (oxidant equivalents to SLPI) for $30 \mathrm{~min}, 25^{\circ} \mathrm{C}$; to insure that the rSLPI exposed to $N$-chlorosuccinimide in vitro was rendered nonfunctional as an inhibitor of NE, the remaining anti-NE activity was evaluated as described above; $(b)$ rSLPI oxidized in this fashion and then exposed to NE (molar ratio SLPI to NE 1:1, $30 \mathrm{~min}, 25^{\circ} \mathrm{C}$ ); and $(c)$ lavage fluid of individuals with cystic fibrosis, a condition known to be associated with a very high burden of neutrophils (24), and hence oxidants and NE on the respiratory surface, i.e., a naturally occurring "in vivo" exposure of SLPI to oxidants and NE. The lavage samples were desalted (PD-10 columns; Pharmacia LKB Biotechnology, Piscataway, $\mathrm{NJ}$ ), lyophilized, and reconstituted in $\mathrm{H}_{2} \mathrm{O}$. The solutions were prepared for electrophoresis by addition of SDS, heated $\left(100^{\circ} \mathrm{C} ; 5 \mathrm{~min}\right)$, and applied to a $12.5 \%$ polyacrylamide gel in the presence of SDS. Immunoblot electrophoretic transfer of proteins onto nitrocellulose was carried out by a variation of the method of Towbin et al. (25) using a rabbit anti-SLPI antibody and an alkaline phosphatase conjugated anti-rabbit IgG antibody (Promega Biotec., Madison, WI). To insure that the SLPI in lavage fluid of the cystic fibrosis patients had been rendered nonfunctional in vivo, the $\alpha 1 \mathrm{AT}$ in the lavage fluid was removed by anti- $\alpha 1 \mathrm{AT}$ affinity chromatography as described above, and the samples analyzed for the amount of SLPI (by ELISA) and anti-NE capacity (as described above).

\section{Results}

Amounts of SLPI in normal lavage fluid. Evaluation of normal lavage fluid demonstrated SLPI levels average $11.0 \pm 1.2 \mathrm{nM}$, with some variation among different individuals (6.6 to 16.6 $\mathrm{nM}$ ) (Fig. 1 $A$ ). The $\alpha 1 \mathrm{AT}$ levels varied over a somewhat larger range (11.9 to $39.6 \mathrm{nM}$; average $24.1 \pm 3.2 \mathrm{nM}$ ). In the study group overall, the ratio of SLPI to $\alpha 1 \mathrm{AT}$ was $0.56 \pm 0.10$, but the range of SLPI and $\alpha 1 \mathrm{AT}$ levels caused the SLPI to $\alpha 1 \mathrm{AT}$ ratio to vary from 0.17 to 1.17 (Fig. $1 B$ ). Assuming that SLPI and $\alpha 1 \mathrm{AT}$ in lavage fluid are equally active against NE, these observations imply that, on the average, SLPI contributes approximately one-third of the total anti-NE protection of the epithelial surface of the respiratory tract.

Function of SLPI in lavage fluid. One approach to estimating the contribution of SLPI to the normal anti-NE capacity in lavage fluid is to capitalize on the knowledge that SLPI can inhibit NE, but not PPE, while $\alpha 1 \mathrm{AT}$ inhibits both. In this regard, using purified rSLPI, NE is inhibited in a dose-dependent manner while the same amounts of rSLPI have no effect on PPE activity (Fig. $2 A$ ). In contrast, $\alpha 1 \mathrm{AT}$ inhibits NE and 

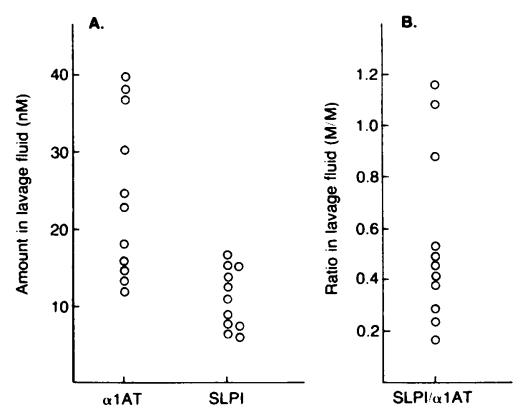

Figure 1. Amounts of SLPI compared with that of $\alpha$ lAT in bronchoalveolar lavage fluid of normal individuals. $(A)$ Amount of SLPI and $\alpha 1 \mathrm{AT}$ lavage fluid. $(B)$ Ratio of the molar concentration of SLPI in lavage fluid to the molar concentration of $\alpha 1 \mathrm{AT}$. Each data point represents one individual evaluated in duplicate.

PPE equally in a dose-dependent fashion (Fig. 2 B). In quantitative terms, rSLPI was $96 \pm 1 \%$ active against NE and $0 \%$ active against PPE, while $\alpha 1 \mathrm{AT}$ was $98 \pm 1 \%$ active against $\mathrm{NE}$ and $100 \pm 2 \%$ active against PPE. This differential inhibitory activity by SLPI and $\alpha 1 \mathrm{AT}$ was used to characterize the antiprotease activity in lavage fluid of normal individuals, a valid approach since SLPI and $\alpha 1 \mathrm{AT}$ are the two major antiproteases in respiratory ELF, and because $\alpha 1 \mathrm{AT}$ in normal individuals is $>95 \%$ active $(10,27-30)$. The average anti-NE capacity of lavage fluid was $27.9 \pm 3.1 \mathrm{nM}$, while the average anti-PPE capacity was $25.3 \pm 3.2 \mathrm{nM}$, i.e., the average anti-PPE capacity is very close to the anti-NE capacity (Fig. $3 \mathrm{~A}$ ). Subtracting the anti-PPE capacity of lavage fluid of each patient from the anti-NE capacity of lavage fluid of the same individual, it is apparent that, on the average, there is only $2.6 \pm 0.8 \mathrm{nM}$ capacity in lavage fluid that can be attributed to a molecule that is capable of inhibiting NE but not inhibiting PPE. In the context that the average SLPI level in the same samples was much higher $(11.1 \pm 1.4 \mathrm{nM})$, and with the knowledge that SLPI is the only known molecule in respiratory ELF with the capacity to inhibit NE but not PPE, it appears the ratio of "functional" SLPI to total SLPI varies, but is always $<0.45$, and with an average of $0.22 \pm 0.04$, i.e., the majority of SLPI does not appear to be functional (Fig. $3 \mathrm{~B}$ ).

As another approach to evaluating the function of SLPI in ELF, $\alpha 1 \mathrm{AT}$ was removed from lavage fluid by affinity chroma-

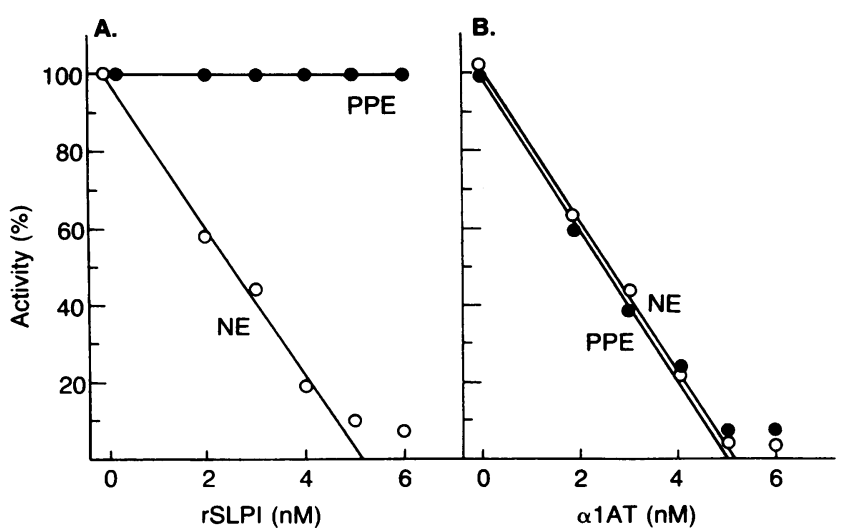

Figure 2. Inhibition of NE and PPE by SLPI compared with $\alpha 1 \mathrm{AT}$. Increasing concentrations of rSLPI or plasma $\alpha 1 \mathrm{AT}$ were incubated with constant concentrations of NE or PPE and the remaining protease activity quantified. Each data point represents the average of triplicate determinations. $(A)$ Anti-NE activity and anti-PPE activity of rSLPI. (B) Anti-NE activity and anti-PPE activity of $\alpha 1 \mathrm{AT}$.

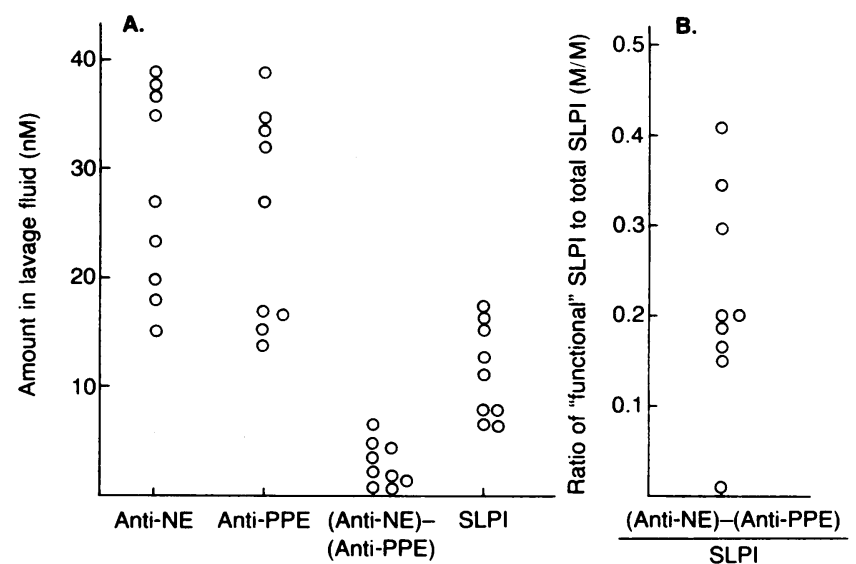

Figure 3. Estimation of the anti-NE capacity of lavage fluid directly attributable to SLPI by comparison of the relative anti-NE capacity to the anti-PPE capacity of lavage fluid. $(A)$ Comparison of the concentration of SLPI and the anti-NE capacity and anti-PPE capacity in respiratory epithelial lining fluid of normal individuals. Shown is anti-NE capacity, anti-PPE capacity, the amount of anti-NE capacity after anti-PPE capacity has been subtracted ("anti-NE - anti-PPE"), and concentration of SLPI in lavage fluid. All data is presented in nM amounts. $(B)$ The molar anti-NE capacity of SLPI to the molar concentration of SLPI in lavage fluid. To estimate the anti-NE capacity in lavage fluid specifically attributable to SLPI, the anti-PPE capacity in lavage fluid was subtracted from the anti-NE capacity. Since $\alpha 1$-antitrypsin inhibits both, but SLPI only NE, the "anti-NE-anti-PPE" capacity estimates the SLPI specific anti-NE capacity (see text). Each data point represents duplicate determinations for a different individual.

tography. Control experiments with solutions of known concentrations of rSLPI and albumin in $0.9 \% \mathrm{NaCl}$ showed that the column procedure did not affect the activity of rSLPI. Before removal, the average SLPI level was $12.6 \pm 3.4 \mathrm{nM} / \mu \mathrm{M}$ albumin, while the average $\alpha 1 \mathrm{AT}$ level was $24.4 \pm 1.6 \mathrm{nM} / \mu \mathrm{M}$ albumin (Fig. $4 A$ ). As before, the anti-NE capacity (28.0 \pm 1.3 $\mathrm{nM} / \mu \mathrm{M}$ albumin) was slightly higher than the anti-PPE capacity $(24.5 \pm 1.2 \mathrm{nM} / \mu \mathrm{M}$ albumin). After removal of $\alpha 1 \mathrm{AT}$, the SLPI level remained the same $(14.0 \pm 3.4 \mathrm{nM} / \mu \mathrm{M}$ albumin, $P$ $>0.1$ compared with before affinity chromatography) (Fig. 4 $B)$. As expected, no $\alpha 1 \mathrm{AT}$ was detectable. Consistent with the concept that $\alpha 1 \mathrm{AT}$ contributes almost all of the anti-NE capacity of lavage fluid and that SLPI does not inhibit PPE, when the $\alpha$ lAT was removed, the anti-NE capacity was reduced to $5.8 \pm 2.3 \mathrm{nM} / \mu \mathrm{M}$ albumin and the anti-PPE capacity was not detectable. Importantly, evaluation of the fluid after removal of $\alpha$ IAT revealed that the anti-NE capacity compared to the SLPI level indicated, as before, that the SLPI was only partially functional (post- $\alpha$ AT removal ratio of anti-NE capacity to level of SLPI $0.38 \pm 0.06$, a value similar to that obtained by indirect analysis)

Aerosolization of rSLPI to sheep. If the SLPI ELISA and the $\alpha 1$ AT ELISA measure the SLPI and $\alpha 1$ AT levels in bronchoalveolar lavage fluid correctly and if the lavage procedure does not destroy the SLPI, deposition of equal concentrations of human rSLPI and human r $\alpha 1 \mathrm{AT}$ in the lung should result in comparable concentrations as assessed by the ELISA. To evaluate this, equal amounts of rSLPI and $\alpha 1 \mathrm{AT}$ ( $50 \mathrm{mg}$ each) were aerosolized to sheep and the rSLPI and r $\alpha 1$ AT levels in bronchoalveolar lavage fluid quantified $1 \mathrm{~h}$ after the aerosol by 


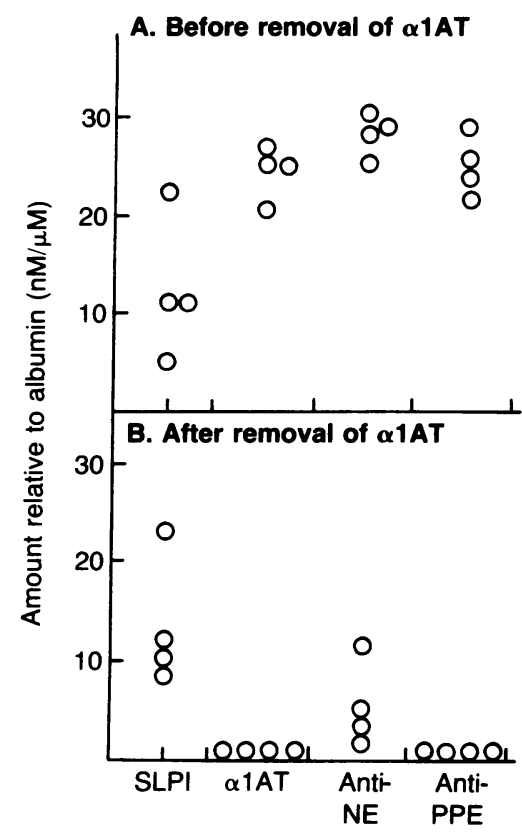
after removal of $\alpha 1 \mathrm{AT}$. The $\alpha 1 \mathrm{AT}$ was removed by affinity chromatography and the fall through was analyzed. Each data point represents a different individual evaluated in duplicate.

ELISA. The molar ratio between rSLPI and $r \alpha 1 \mathrm{AT}$ in the aerosolized solution was 3.75 (molecular mass rSLPI $12 \mathrm{kD}$, r $\alpha 1 \mathrm{AT}$ $45 \mathrm{kD}$ ). The molar ratio of the levels of rSLPI and $\mathrm{r} \alpha 1 \mathrm{AT}$ in lavage fluid after the aerosol was nearly identical $(3.55 \pm 0.31 ; P$ $>0.5$ ) (Fig. $5 A$ ). To assess whether SLPI was inactivated by the lavage procedure, rSLPI $(50 \mathrm{mg})$ was administered to sheep by
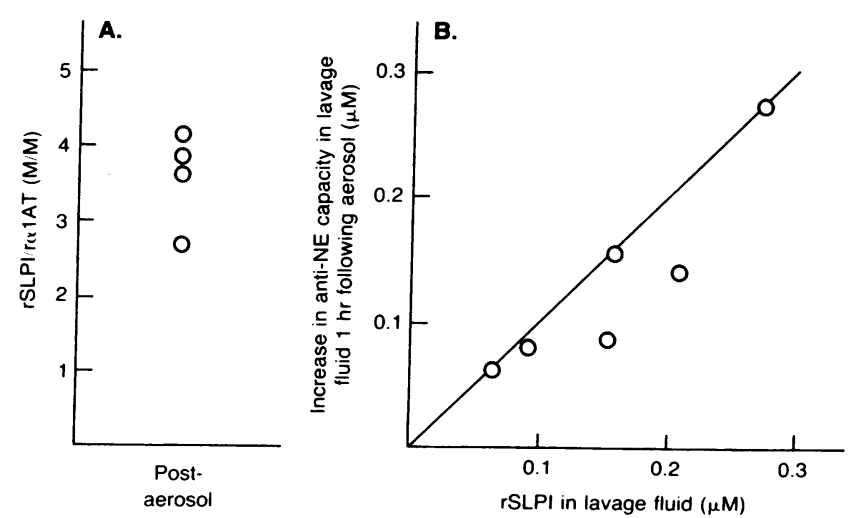

Figure 5. Demonstration that fully functional SLPI can be recovered from the epithelial surface of the lung by bronchoalveolar lavage. As a control for the recovery methods used, rSLPI was aerosolized to sheep alone or in combination with $r \alpha 1 \mathrm{AT}$. $(A)$ rSLPI and $\mathrm{r} \alpha 1 \mathrm{AT}$ were aerosolized simultaneously and the rSLPI and r $\alpha 1 \mathrm{AT}$ levels in lavage fluid quantified. The molar ratio between the concentration of rSLPI and $r \alpha 1 \mathrm{AT}$ in the aerosolized solution was 3.75. Shown is the ratio in lavage fluid after the aerosol ("post-aerosol"). (B) rSLPI was aerosolized alone to sheep and the concentration of rSLPI in lavage fluid after the aerosol compared with the increase in the antiNE capacity of the fluid. The starting material was $96 \pm 1 \%$ functional; if all recovered rSLPI was as functional as the starting material, all data would be on the diagonal line. For both panels, each data point represents one animal with values evaluated in duplicate.

aerosol. $1 \mathrm{~h}$ later, ELF was recovered by lavage and the rSLPI level was quantified. To determine if the amount of rSLPI present corresponded to the increase in anti-NE capacity of the fluid, the anti-NE capacity $1 \mathrm{~h}$ after aerosolization was compared with the anti-NE capacity of the fluid before aerosolization (i.e., the anti-NE capacity due to the rSLPI after the endogenous anti-NE capacity was subtracted). This analysis demonstrated a direct correlation between the concentration of rSLPI in removed ELF and the increase in the anti-NE capacity ( $r$ $=0.89$; Fig. $5 \mathrm{~B}$ ). Thus the SLPI ELISA and the $\alpha 1 \mathrm{AT}$ ELISA can accurately quantify the respective molecules in lavage fluid and the anti-NE activity of SLPI is not compromised by the lavage procedure.

Evaluation of SLPI in normal lavage fluid by size exclusion chromatography. One hypothesis to explain the observed dysfunction of SLPI in normal lavage fluid is that the majority of the SLPI has complexed with another molecule such as NE, its natural target. To evaluate this, normal lavage fluid was evaluated by size exclusion HPLC in physiologic conditions. However, the SLPI in lavage fluid chromatographed in a position identical to uncomplexed SLPI (12 kD), with no SLPI detectable at a higher molecular mass (such as $41 \mathrm{kD}$, as would be expected from a SLPI-NE complex) (Fig. 6). Thus, it appears that SLPI on the epithelial surface of the normal respiratory tract is not complexed to NE.

Evaluation of the effect of oxidation on SLPI. To evaluate the concept that SLPI on the normal epithelial surface might be inactivated by oxidants in vivo and thus be unable to interact with NE should it be exposed to it, analytic methods were developed that could detect oxidized SLPI exposed to NE (Figs. 7, 8). In this regard, exposure of rSLPI to increasing amounts of oxidant rendered it unable to inhibit NE (Fig. $7 \mathrm{~A}$ ). Evaluation of the form of rSLPI demonstrated that while initially intact (Fig. 8, lane 1), and remaining intact when exposed to NE alone (lane 2), or oxidants alone (lane 3 ), the combination of oxidants + NE causes fragmentation of the rSLPI (lane 4). A possible in vivo correlate of this experiment is cystic fibrosis, a condition where SLPI on the epithelial surface is likely exposed

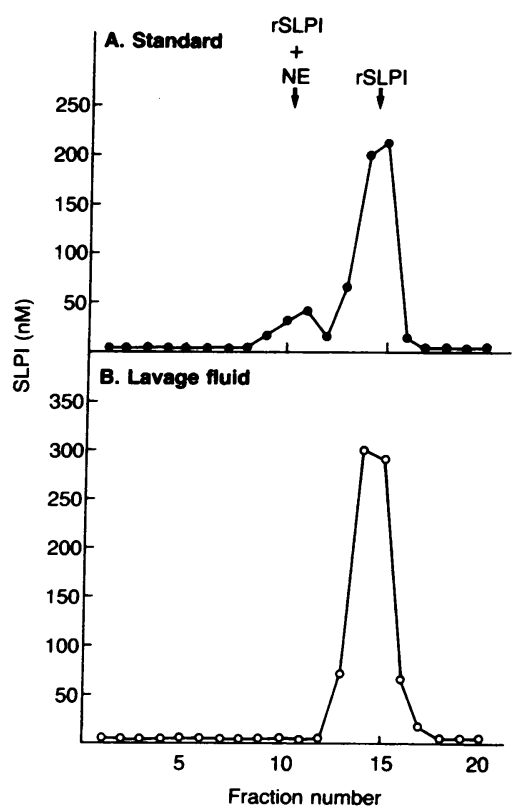

Figure 6. Form of SLPI in normal respiratory epithelial lining fluid as evaluated by size exclusion HPLC of normal bronchoalveolar lavage fluid. Chromatographed samples were analyzed using an enzyme linked immunoassay. $(A)$ Standard of rSLPI that had been incubated with NE. The positions of rSLPI alone and the complex of rSLPI and $\mathrm{NE}$ are indicated. $(B)$ Bronchoalveolar lavage fluid of a normal individual. 

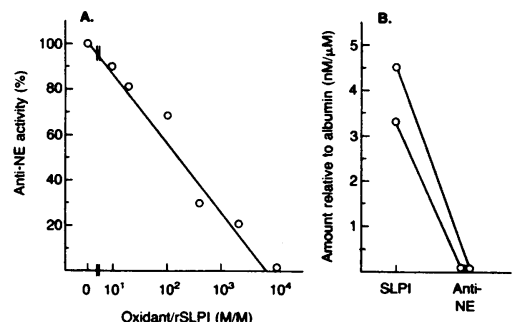

Figure 7. Inactivation of SLPI. $(A)$ rSLPI was inactivated in vitro by oxidation with varying amounts of $N$ chlorosuccinimide and the remaining anti-NE activity was quantified. Shown is the average of duplicate determinations. (B) As an in vivo model for inactivation of SLPI, lavage fluid of cystic fibrosis patients was evaluated for the presence of SLPI and anti-NE capacity after $\alpha 1 \mathrm{AT}$ was removed from the lavage fluid by affinity chromatography. The SLPI level and the anti-NE capacity were quantified in the fall through compared with albumin.

to a heavy burden of both oxidants and NE (24). As expected, evaluation of the amount of SLPI in lavage fluid in cystic fibrosis revealed SLPI was present, but completely inactive as an inhibitor of NE (Fig. 7 B). Consistent with this concept, Western analysis demonstrated the SLPI in cystic fibrosis lavage fluid was fragmented (Fig. 8, lane 6). In contrast, however, parallel analysis of lavage fluid from normals demonstrated the SLPI was intact. Thus, while it is possible that the interaction of SLPI observed in normals results from in vivo oxidation, based upon the evaluation of SLPI from cystic fibrosis individuals, current evidence argues that it is not in vivo oxidation + exposure to NE.

Concentration of SLPI in upper versus lower respiratory tract epithelial lining fluid. To evaluate the anatomic distribution of SLPI in ELF of the normal respiratory tract, normals were lavaged sequentially, the recovered aliquots collected separately and the concentrations of SLPI assessed in each sample. As expected, the concentrations of SLPI in the first sequential sample representing upper airways ELF were significantly higher $(8.7 \pm 0.8 \mu \mathrm{M})$ than in the last sequential sample representing lower respiratory tract $\operatorname{ELF}(0.6 \pm 0.1 \mu \mathrm{M}, P<0.001)$ (Fig. $9 \mathrm{~A}$ ). Comparing the concentrations of SLPI with the concentrations of $\alpha 1 \mathrm{AT}$ in each sequential sample, it was apparent that SLPI dominated in upper respiratory tract ELF, while $\alpha 1 \mathrm{AT}$ dominated in lower respiratory tract ELF (molar ratio of SLPI to $\alpha 1 \mathrm{AT}$ in aliquot No. 1: $1.9 \pm 0.4$ versus aliquot No. 5: $0.3 \pm 0.1 ; P<0.005)$ (panel $B)$.

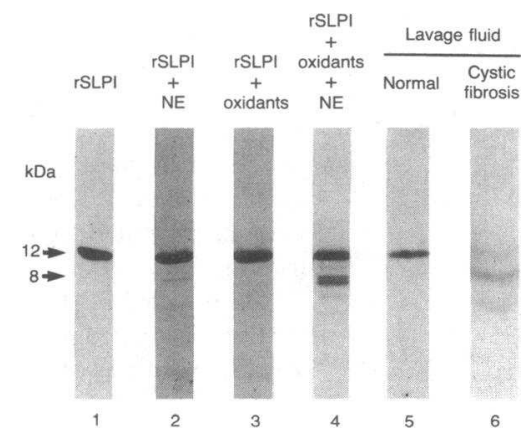

Figure 8. Western blot analysis of SLPI in bronchoalveolar lavage fluid. Shown is the antiSLPI immunoblot evaluation of: lane 1 , rSLPI standard; lane 2, rSLPI incubated with NE; lane 3, oxidized rSLPI; lane 4, oxidized rSLPI incubated with NE; lane 5 , bronchoalveolar lavage fluid of a normal

individual; and lane 6, bronchoalveolar lavage fluid of an individual with cystic fibrosis. The molecular mass $(\mathrm{kD})$ of the major bands is indicated.
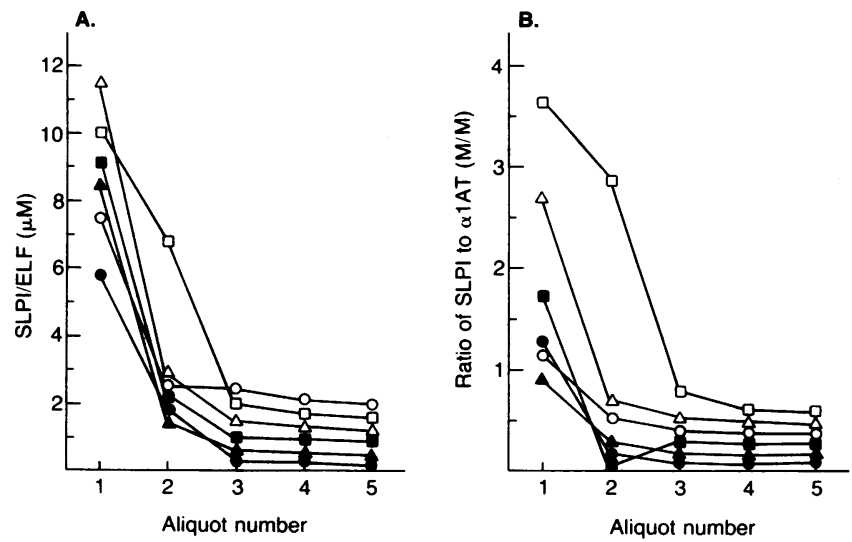

Figure 9. Amounts of SLPI in upper versus lower respiratory tract ELF. Normals (each indicated by a different symbol) were lavaged sequentially and the SLPI and $\alpha 1 \mathrm{AT}$ concentrations were measured in each of the aliquots. (A) Levels of SLPI in ELF. (B) Ratio of the molar concentration of SLPI in ELF to the molar concentration of $\alpha 1 \mathrm{AT}$.

\section{Discussion}

It has been recognized for some time that secretory leukoprotease inhibitor is present in cells lining the tracheobronchial tree (7-9) and in the epithelial lining fluid lining the respiratory tract (10-13). In the context that SLPI is an excellent inhibitor of neutrophil elastase, these observations have led to the conclusion that SLPI plays a role in the defense of the respiratory epithelial surface $(31,32)$. This study presents the surprising observation that $\sim 67 \%$ of this respiratory surface SLPI is unable to inhibit its natural substrate, neutrophil elastase. While the mechanisms responsible for this are unknown, the SLPI in respiratory ELF is intact, and not complexed to other molecules, such as neutrophil elastase. These observations support the concept that SLPI plays, at most, a minor role in protecting the lower respiratory tract from NE. However, despite the fact that only a small proportion of the SLPI is functional, sequential lavage studies demonstrated that the concentration in upper airways ELF is sufficiently high so that it is possible that SLPI does play a role in providing some anti-NE defense for the epithelial surface of the trachea and the bronchial tree.

Evaluation of SLPI. Together, a number of factors strongly argue that the observation that two-thirds of the SLPI recovered from the respiratory epithelium is nonfunctional is a true reflection of the state of SLPI in respiratory ELF in normal humans in vivo.

First, to avoid possible artifacts introduced by local disease processes and/or cigarette smoke, we included only healthy, nonsmoking volunteers in the study group. In such individuals, using similar methods to evaluate $\alpha 1 \mathrm{AT},>95 \%$ of the $\alpha 1 \mathrm{AT}$ in lavage fluid is fully functional, capable of inhibiting NE.

Second, the measurements concerning levels and function were done in unconcentrated lavage samples, thus obviating artifacts such as oxidation during concentration by pressure filtration.

Third, the use of recombinant DNA methods to produce and purify a functional rSLPI molecule identical to naturally occurring SLPI provided a "gold standard" to compare to SLPI produced in vivo in normal individuals and in fluids recovered 
from the respiratory epithelial surface. Whereas the rSLPI standard was $96 \%$ active against neutrophil elastase, approximately two-thirds of the SLPI recovered from the normal human lung was unable to inhibit neutrophil elastase. Further, to make the assays comparable, the standards for the NE and PPE activity were titrated against the same pure $\alpha 1 \mathrm{AT}$ standard (20). The accuracy of the assays for the determination of the anti-NE and the anti-PPE capacity was confirmed by the fact that the $\alpha 1 \mathrm{AT}$ standard inhibited NE and PPE on a mole per mole basis, whereas the rSLPI standard did so with NE, but was unable to inhibit PPE.

Fourth, aerosolization of rSLPI (alone, or in combination with $\mathrm{r} \alpha \mathrm{AT}$ ) to sheep demonstrated that functional rSLPI deposited on the epithelial surface of the lung could be recovered by lavage in amounts and function identical to that which was deposited, i.e., the impotence of SLPI observed in lavage fluid from normal individuals is not an artifact of the methods used for its recovery.

Finally, in the context that $95 \%$ of $\alpha 1 \mathrm{AT}$ in respiratory ELF is functional, but only one-third of the SLPI is functional, even though the molar ratio of SLPI to $\alpha 1 \mathrm{AT}$ present in lavage fluid recovered from the respiratory surface is 0.56 , the estimated molar ratio of functional SLPI to functional $\alpha 1 \mathrm{AT}$ is only 0.16 . This value is close to the estimates of the total non- $\alpha 1 \mathrm{AT}$ contribution to the total anti-NE capacity of lavage fluid in the circumstance of the Null-Null phenotype of $\alpha 1 \mathrm{AT}$ deficiency, i.e., a circumstance where there is no $\alpha 1 \mathrm{AT}$ produced because of a mutation in the $\alpha 1 \mathrm{AT}$ gene, and thus $\alpha 1 \mathrm{AT}$ cannot contribute to the anti-NE capacity of the fluid recovered from the lung (33).

Possible mechanisms causing inactivation of SLPI in vivo. Although the data in this study demonstrates that the majority of the SLPI in respiratory ELF of normals is inactive, it is not clear why this occurs. From the available data, it is reasonable to conclude that the SLPI is intact, is not complexed to NE (or other molecules), and has not been exposed to oxidants and NE in vivo, as is the case in cystic fibrosis.

Consistent with the anatomical distribution of SLPI producing cells in respiratory epithelium, there is a concentration gradient for SLPI from the upper to the lower respiratory tract (11-13). Since SLPI is susceptible to oxidation, it is conceivable that SLPI on the epithelial surface is oxidized in vivo by oxidants, for instance, from inflammatory cells present on the epithelial surface or inhaled oxidants in ambient air. If this is the case, it would have to be put in the context that $\alpha 1 \mathrm{AT}$ in normal respiratory epithelial fluid is not oxidized $(10,27-30)$. With available methods, it is not possible to recover sufficient SLPI and $\alpha 1$ AT from upper airway ELF to determine if inactivated SLPI in lavage fluid is derived primarily from SLPI in upper airway ELF, i.e., there could be a gradient down the tracheobronchial tree, such that SLPI in upper airway ELF is completely inactivated due to a higher burden of oxidants in this location compared with SLPI in the lower respiratory tract that is relatively more functional. Consistent with this concept, there is a higher concentration of neutrophils per microliter of upper respiratory tract ELF than in lower respiratory tract ELF (34). In the context that incubation of SLPI with stimulated human polymorphonuclear leukocytes results in a rapid loss of anti-NE activity via oxidative mechanisms (35), it is conceivable that neutrophils normally present in the upper airways are responsible for the inactivation of SLPI in vivo. Further, it is likely that upper airway SLPI is exposed to a higher concentra- tion of inhaled oxidants in ambient air, a concept consistent with studies showing the activity of SLPI against trypsin was markedly reduced in tracheal aspirates from smokers compared with that of nonsmokers (36).

Relevance of SLPI to the anti-neutrophil defenses of the lung. Despite the fact the most of the SLPI recovered from the normal respiratory surface is inactive, this does not mean it does not play a role in defending the lung against NE. It is conceivable that SLPI plays a first line role, particularly in the upper airways where the concentrations of SLPI are highest (10-13). Although there is no evidence demonstrating a selective advantage for SLPI or $\alpha 1 \mathrm{AT}$ in the inactivation of NE when both molecules are present, it is likely that within tissues SLPI and $\alpha 1 \mathrm{AT}$ compartmentalize differently. In this regard, SLPI has a much lower molecular mass ( $12 \mathrm{kD}$ compared with $52 \mathrm{kD}$ for $\alpha 1 \mathrm{AT}$ ), and a much higher isoelectric point (pI) (10, compared with 4.7 for $\alpha 1 \mathrm{AT}$ ). The SLPI pI is close to that of NE, suggesting the SLPI might compartmentalize in a similar fashion to its natural substrate.

Even if the role of SLPI in defending the respiratory surface is less than the numbers of SLPI molecules may suggest, this does not mean that SLPI might not be useful therapeutically in helping to augment the anti-NE defenses of the lung. In this regard, when SLPI is deposited in respiratory ELF, it remains functional at least in the short term (37). In the context that SLPI and $\alpha 1 \mathrm{AT}$ are approximately equal as inhibitors of NE, SLPI is one alternative to augment anti-NE defenses in hereditary and/or acquired disorders of the lung where the lung is vulnerable to attack by NE.

\section{References}

1. Grutter, M. G., G. Fendrich, R. Huber, and W. Bode. 1988. The $2.5 \AA$ $\mathrm{X}$-ray crystal structure of the acid-stable proteinase inhibitor from human mucous secretions analysed in its complex with bovine $\alpha$-chymotrypsin. $E M B O$ (Eur. Mol. Biol. Organ.) J. 7:345-351.

2. Fritz, H. 1988. Human mucus proteinase inhibitor (human mpi). Biol. Chem. Hoppe-Seyler. 369 S:79-82.

3. Hochstasser, K., R. Reichert, S. Shwarz, and E. Werle. 1972. Isolierung und Charakterisierung eines Proteaseninhibitors aus menschlichem Bronchialsekret. Hoppe-Seyler's Z Physiol. Chem. 353:221-226.

4. Seemüller, U., M. Arnhold, H. Fritz, K. Wiedenmann, W. Machleidt, R. Heinzel, H. Appelhans, H.-G. Gassèn, and F. Lottspeich. 1986. The acid-stable proteinase inhibitor of human mucous secretions (HUSI-I. anti-leukoprotease). FEBS (Fed. Eur. Biol. Soc.) Lett. 199:43-48.

5. Thompson, R. C., and K. Ohlsson. 1986. Isolation properties, and complete amino acid sequence of human secretory leukocyte protease inhibitor, a potent inhibitor of leukocyte elastase. Proc. Natl. Acad. Sci. USA. 83:6692-6696.

6. Gauthier, F., U. Fryksmark, K. Ohlsson, and J. G. Bieth. 1982. Kinetics of the inhibition of leukocyte elastase by the bronchial inhibitor. Biochem. Biophys. Acta. 700:178-183.

7. De Water, R., L. N. A. Williams, G. N. P. Van Muijen, C. Franken, J. A. M. Fransen, J. H. Dijkman, and J. A. Kramps. 1986. Ultrastructural localization of bronchial antileukoprotease in central and peripheral human airways by a goldlabeling technique using monoclonal antibodies. Am. Rev. Respir. Dis. 133:882890.

8. Fryksmark, U., K. Ohlsson, A. Polling, and H. Tegner. 1982. Distribution of antileukoprotease in upper respiratory mucosa. Ann. Otol. Rhinol. \& Laryngol. 91:268-271.

9. Mooren, H. W. D., J. A. Kramps, C. Franken, C. J. L. M. Meijer, J. A. Dijkan. 1983. Localization of a low-molecular-weight bronchial protease inhibitor in the peripheral human lung. Thorax. 38:180-138.

10. Boudier, C., A. Pelletier, A. Gast, J. M. Tournier, G. Pauli, and J. G. Bieth. 1987. The elastase inhibitory capacity and the $\alpha_{1}$-proteinase inhibitor and bronchial inhibitor content of bronchoalveolar lavage fluids from healthy subjects. Biol. Chem. Hoppe-Seyler. 368:981-990.

11. Kramps, J. A., C. Franken, and J. H. Dijkman. 1988. Quantity of anti-leukoprotease relative to $\alpha 1$-proteinase inhibitor in peripheral airspace of the human lung. Clin. Sci. 75:351-353.

12. Morrison, H. M., J. A. Kramps, J. H. Dijkman, and R. A. Stockley. 1986. Comparison of concentrations of two proteinase inhibitors, porcine pancreatic 
elastase inhibitory capacity, and cell profiles in sequential bronchoalveolar lavage samples. Thorax. 41:435-441.

13. Smith, S. F., A. Guz, A. J. Winning, N. T. Cooke, G. H. Burton, and T. D. Tetley. 1988. Comparison of human lung surface protein profiles from the central and peripheral airways sampled using two regional lavage techniques. Eur. $R \boldsymbol{e}$ spir. J. 1:792-800.

14. Rennard, S. I., G. Basset, D. Lecossier, K. M. O'Donnell, P. Pinkston P. G. Martin, and R. G. Crystal. 1984. Estimation of volume of epithelial lining fluid recovered by lavage using urea as marker of dilution. J. Appl. Physiol. 60:532-538.

15. Kramps, J. A., C. Franken, and J. H. Dijkman. 1984. ELISA for quantitative measurement of low-molecular-weight bronchial protease inhibitor in human sputum. Am. Rev. Respir. Dis. 129:959-963.

16. Eisenberg, S. P., K. K. Hale, P. Heimdal, and R. C. Thompson. 1990. Location of the protease inhibitory region of secretory leukocyte-protease inhibitor. J. Biol. Chem. In press.

17. Miller, K. W., R. J. Evans, S. P. Eisenberg, and R. C. Thompson. 1989 Secretory leukocyte protease inhibitor binding to mRNA and DNA as a possible cause of toxicity to Escherichia coli. J. Bacteriol. 171:2166-272.

18. Kohno, T., D. F. Carmichael, A. Sommer, and R. C. Thompson. 1990. Refolding of recombinant proteins. Methods Enzymol. In press.

19. Hubbard, R. C., M. A. Casolaro, M. Mitchell, S. E. Sellers, F. Arabia M. A. Matthay, and R. G. Crystal. 1989. Fate of aerosolized recombinant DNAproduced $\alpha 1$-antitrypsin: use of the epithelial surface of the lower respiratory tract to administer proteins of therapeutic importance. Proc. Natl. Acad. Sci. USA 86:680-684.

20. Ogushi, F., C. A. Fells, R. C. Hubbard, S. D. Straus, and R. G. Crystal 1987. Z-type $\alpha 1$-antitrypsin is less competent than M1-type $\alpha 1$-anti-trypsin as an inhibitor of neutrophil elastease. J. Clin. Invest. 80:1366-1374.

21. Beatty, K., J. Bieth, and J. Travis. 1980. Kinetics of association of serine proteinase with native and oxidized $\alpha$-1-proteinase inhibitor and $\alpha$-1-antichymotrypsin. J. Biol. Chem. 255:3931-3934.

22. Smith, R. M., L. D. Traber, D. L. Traber, and R. G. Spragg. 1989. Pulmonary deposition and clearance of aerosolized alpha-1-proteinase inhibitor administered to dogs and to sheep. J. Clin. Invest. 84:1145-1154.

23. Wewers, M. D. M. A Casolaro, S. E. Sellers, S. A. Swayze, K. M. McPail, J. T. Wittes, and R. G. Crystal. 1987. Replacement therapy for alpha 1 antitrypsin deficiency. N. Engl. J. Med. 316:1055-1062.

24. Goldstein, W., and G. Döring. 1986. Lysomal enzymes from polymorphonuclear leukocytes and proteinase inhibitors in patients with cystic fibrosis Am. Rev. Respir. Dis. 134:49-56.
25. Towbin, H., T. Staehelin, and J. Gordon. 1979. Electrophoretic transfer of proteins from polyacrylamide gels to nitrocellulose sheets: procedure and some applications. Proc. Natl. Acad. Sci. USA. 76:4350-4354.

26. Stockley, R. A. 1988. Chronic bronchitis: the antiproteinase/proteinase balance and the effect of infection and corticosteroids. Clin. Chest Med. 9:643656.

27. Padrines, M., Schneider-Pozzer, and J. G. Bieth. 1989. Inhibition of neutrophil elastase by alpha-1-proteinase inhibitor oxidized by activated neutrophils. Am. Rev. Respir. Dis. 139:783-790.

28. Boudier, C., A. Pelletier, G. Pauli, and J. G. Bieth. 1983. The functiona activity of $\alpha 1$-proteinase inhibitor in broncheoalveolar lavage fluids from healthy human smokers and non-smokers. Clin. Chem. Acta. 132:309-315.

29. Gadek, J. E., G. A. Fells, and R. G. Crystal. 1979. Cigarette smoking induces functional antiproteinase deficiency in the lower respiratory tract of humans. Science (Wash. DC). 206:1315-1316.

30. Wewers, M. D., Herzyk D. J., Gadek J. E. 1989. Comparison of smoker and nonsmoker lavage fluid for the rate of association with neutrophil elastase. Am. J. Respir. Cell. Mol. Biol. 1:423-429.

31. Hutchinson, D. C. S. 1987. The role of protease and antiprotease in bronchial secretions. Eur. J. Respir. Dis. 71(Suppl.):78-85.

32. Bieth, J. G. 1986. Elastases: Catalytic and biological properties. In Regulation of matrix accumulation. Academic Press, Inc., New York 217-320.

33. Wewers, M. D., A. C. Casolaro, and R. G. Crystal. 1987. Comparison of alpha 1-antitrypsin levels and anti-neutrophil elastase capacity of blood and lung in a patient with the alpha 1-antitrypsin phenotype null-null before and during alpha 1-antitrypsin augmentation therapy. Am. Rev. Respir. Dir. 135:539-543.

34. Rennard S. I. M. Ghafouri, A. B. Thompson, J. Linder, W. Vaughan, K. Jones, R. F. Ertl, K. Christensen, A. Prince, M. G. Stahl, and R. A. Robbins 1990. Fractional processing of sequential bronchoalveolar lavage to separate bronchial and alveolar samples. Am. Rev. Respir. Dis. 141:208-217.

35. Kramps, J. A., Ch. Van Twisk, E. C. Klasen, and J. H. Dijkman. 1988. Interactions among stimulated human polymorphonuclear leucocytes, released elastase and bronchial antileucoprotease. Clin. Sci. 75:53-62.

36. Carp, H., and A. Janoff. 1980. Inactivation of bronchial mucous proteinase inhibitor by cigarette smoke and phagocyte-derived oxidents. Exp. Lung Res. 1:225-237.

37. Vogelmeier, C., R. Buhl, R. F. Hoyt, E. Wilson, G. A. Fells, R. C. Hubbard, H. P. Schneri, R. C. Thompson, and R. G. Crystal. 1990. Aerosolization of recombinant secretory leukoprotease inhibitor as a strategy to augment the antineutrophil elastase protective screen of the pulmonary epithelial surface. $\mathrm{Am}$. Rev. Respir. Dis. 141: 47a. (Abstr.) 